\title{
Approximately Quantized Thermal Hall Effect of Chiral Liquids Coupled to Phonons
}

\author{
Yuval Vinkler-Aviv and Achim Rosch \\ Institute for Theoretical Physics, University of Cologne, 50937 Cologne, Germany
}

(Received 4 June 2018; published 1 August 2018)

\begin{abstract}
The recent observation of a half-integer quantized thermal Hall effect in $\alpha-\mathrm{RuCl}_{3}$ is interpreted as a unique signature of a chiral spin liquid with a Majorana edge mode. A similar quantized thermal Hall effect is expected in chiral topological superconductors. The unavoidable presence of gapless acoustic phonons, however, implies that, in contrast to the quantized electrical conductivity, the thermal Hall conductivity $\kappa_{x y}$ is never exactly quantized in real materials. Here, we investigate how phonons affect the quantization of the thermal conductivity, focusing on the edge theory. As an example, we consider a Kitaev spin liquid gapped by an external magnetic field coupled to acoustic phonons. The coupling to phonons destroys the ballistic thermal transport of the edge mode completely, as energy can leak into the bulk, thus drastically modifying the edge picture of the thermal Hall effect. Nevertheless, the thermal Hall conductivity remains approximately quantized, and we argue that the coupling to phonons to the edge mode is a necessary condition for the observation of the quantized thermal Hall effect. The strength of this edge coupling does, however, not affect the conductivity. We argue that for sufficiently clean systems the leading correction to the quantized thermal Hall effect, $\Delta \kappa_{x y} / T \sim \operatorname{sign}(\mathrm{B}) T^{2}$, arises from an intrinsic anomalous Hall effect of the acoustic phonons due to Berry phases imprinted by the chiral (spin) liquid in the bulk. This correction depends on the sign but not the amplitude of the external magnetic field.
\end{abstract}

DOI: 10.1103/PhysRevX.8.031032

Subject Areas: Condensed Matter Physics, Strongly Correlated Materials, Topological Insulators

\section{INTRODUCTION}

Quantum Hall effects are prime examples showing how topological properties of matter lead to a precise quantization of physical observables. The integer and fractional quantum Hall effects are characterized by quantized Hall conductivity $\sigma_{x y}=(n / m)\left[e^{2} /(2 \pi \hbar)\right]$, with integer $n, m$, and by a vanishing longitudinal conductivity $\sigma_{x x}=0$. A quantum Hall effect in the charge sector is usually also associated with a thermal quantum Hall effect. The latter can, however, also occur separately. Both for topological superconductors and insulators [1-3] and for certain chiral spin liquids (e.g., described by the Kitaev model [4]), a quantized thermal Hall effect, $(1 / T) \kappa_{x y}=(n / m)\left[\left(\pi k_{B}^{2}\right) /(6 \hbar)\right]$, has been predicted in close analogy to the (anomalous) Hall effect in two-dimensional conductors.

Recently, a spectacular experiment by Kasahara et al. [5] reported the discovery of a half-integer quantized thermal Hall effect in $\alpha-\mathrm{RuCl}_{3}$. This material is believed to be

Published by the American Physical Society under the terms of the Creative Commons Attribution 4.0 International license. Further distribution of this work must maintain attribution to the author(s) and the published article's title, journal citation, and DOI. approximately described by a Kitaev model on a honeycomb lattice [6-27]. While it orders magnetically at zero magnetic field, Kasahara et al. identified a range of fields where the magnetic order is apparently suppressed and a gapped spin liquid characterized by a quantized thermal Hall effect emerges. Such a phase has been predicted by Kitaev [4] in the idealized honeycomb Kitaev model. The two-dimensional honeycomb Kitaev model is an example of a frustrated spin liquid that can accurately be described by Majorana fermions coupled to a $\mathbb{Z}_{2}$ gauge field. In the presence of a magnetic field, a gap opens up in the bulk and chiral edge modes of Majorana fermions arise at the boundaries of the sample. The latter carries heat and leads to a quantized thermal Hall effect, $(1 / T) \kappa_{x y}=\frac{1}{2}\left[\left(\pi k_{B}^{2}\right) /(6 \hbar)\right]$, precisely as observed experimentally.

There is a major difference between the electric and the thermal Hall effect when realized in real material: In all solids, phonons provide a thermal conduction mechanism, leading to a finite heat conductivity $\kappa_{x x}$, typically with $\kappa_{x x} \gg \kappa_{x y}$. In contrast, a hallmark of the quantized electrical Hall response is a vanishing bulk conductivity. Theoretical models predicting a thermal quantum Hall effect usually ignore the presence of phonons (thus assuming or concluding that $\kappa_{x x}=0$ ) and, sometimes, it is argued that the 
coupling of phonons will prohibit the observation of a quantized thermal Hall effect.

There are two complementary ways to understand quantum Hall effects: the bulk and the edge perspective. From the first point of view, Hall effects are bulk phenomena. The thermal conductivity can be calculated, e.g., from an appropriate Kubo formula in a large system with periodic boundary conditions. An alternative and more intuitive point of view explains quantum Hall effects by the presence of chiral conducting channels that exist at the edge of the sample. The presence of the edge channels is enforced and protected by the (insulating) bulk theory. The resulting bulk-edge correspondence is a manifestation of the holographic principle and intimately connected to topological field theories and the physics of anomalies. Charge anomalies reflect that a change of charge in the bulk of the system (e.g., when the magnetic field is changed) can only occur at the gapless edge of a system. The change of magnetic field thereby induces an electric field, which pumps charges into chiral edge modes. For the thermal Hall effect, similar arguments exist where the electromagnetic field is replaced by a (fictitious) gravitational field $[1,3,28-$ 35], i.e., a change of the metric, which leads to pumping of energy instead of charge. When discussing anomalies, one usually assumes a gapped bulk, and it is a priori not fully clear how the presence of gapless phonons will affect the result and the quantization of the Hall effect.

Edge theories of the Hall effect use the fact that chiral modes can transport charge or energy without dissipation. The latter is, however, not valid in the presence of phonons, which destroy ballistic edge transport. Heat leaks out from the edge into the bulk. Therefore, recent measurements by the Weizmann group [36,37] of the thermal transport of (fractional) quantum Hall edge channels had to use a refined setup that involved mesoscopic measurements of the temperature of edge channels using noise spectroscopy. With this setup, the authors could minimize the leakage of heat due to phonons. In contrast, the experiments in $\alpha-\mathrm{RuCl}_{3}$ by Kasahara et al. [5] used macroscopic contacts and operated in the opposite regime, where edge states and phonons are expected to equilibrate. We will show that, counterintuitively, the phonon leakage of heat actually helps to observe an approximately quantized thermal Hall effect. We will discuss both two-dimensional models and three-dimensional systems described, e.g., by weakly coupled layers of Kitaev models in the presence of threedimensional phonons.

It is obvious that, in the presence of phonons, a true quantization of the thermal Hall effect is not possible. Here, "true quantization" is defined as an effect that becomes more and more quantized with exponential precision when the temperature is lowered and the size of the system is increased. Any insulator in a magnetic field (or in a ferromagnetic state) is characterized by a finite thermal Hall effect at finite temperatures, which is not exponentially suppressed with temperature but expected to vanish with a power law of $T$. Nevertheless, the observed phonon thermal Hall effects of the insulator are typically tiny. Very few experiments [38] exist; the first one, from 2005 [39], reports a Hall angle of only $10^{-4}$ rad per Tesla of applied field. Several mechanisms can explain phonon Hall effects. In the literature, a Raman-type interaction between phonons and large spins [40,41], effects of Berry curvature of phonon bands $[42,43]$, and a resonant skew scattering of phonons [44] have been discussed.

In the following, we will discuss the approximate quantization of the thermal Hall effect from the viewpoint of the edge theory and develop a theory of an anomalous thermal Hall effect based on the coupling of phonons to the Kitaev model.

\section{THERMAL HALL EFFECT}

\section{A. Quantized ballistic Hall effect in the absence of phonons}

We begin our discussion by recalling how the thermal Hall effect is described in terms of a chiral edge mode in the absence of phonons, when the bulk is a thermal insulator. In this case, thermal transport is only possible along a chiral edge mode. Figure 1(a) shows a sketch of such a situation. The chiral edge mode carries a finite heat current, which is a function of temperature, $J_{e}(T)$. As in the absence of bulk (a)

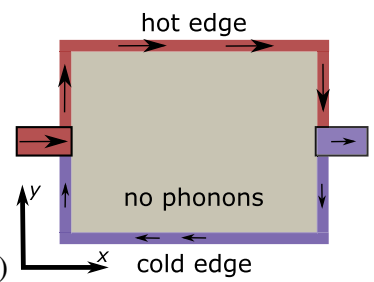

(c)

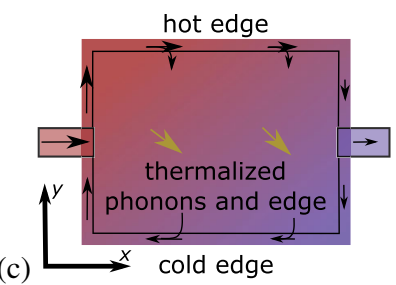

(b)

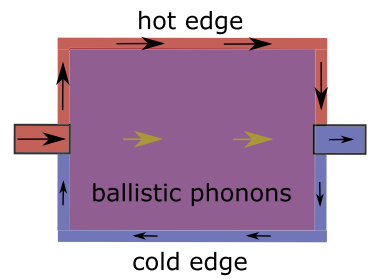

hot edge

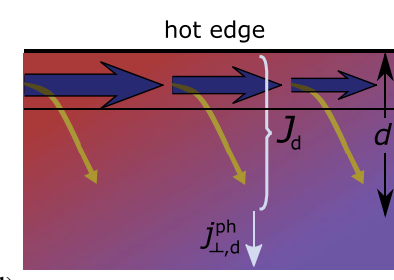

(d)
FIG. 1. Sketch of heat currents (arrows) and local temperatures (color scale) in the presence of chiral edge channels. In the absence of phonons, panel (a), heat is transported ballistically by the edge channel, resulting in a quantization of $\kappa_{x y} / T$. Panel (b) shows a regime where the sample size is smaller than the phonon-edge scattering length, such that the edge states and phonons have different temperatures. No quantization of $\kappa_{x y}$ is expected in this case. In the opposite limit, panel (c), the edge channel and the phonons have the same local temperatures. The temperature gradient imprinted by the phonons onto the edge channel implies that a quantized heat current is injected from the edge mode into the bulk phonons, as shown in panel (d). This leads to quantization of $\kappa_{x y}$ with high precision. 
modes, the energy density at the edge $E_{e}(\boldsymbol{r}, t)$ is conserved, $\partial_{t} E+\nabla_{\|} J_{e}=0$, the heat current is constant in the stationary state, $\nabla_{\|} J_{e}=0$. Here, $\nabla_{\|}$is a spatial derivative parallel to the edge of the system. Therefore, the temperatures along the top and bottom side of the sample, $T_{\text {top }}$ and $T_{\text {bot }}$, are constant and the total energy current through the system is given by $J_{T}=J_{e}\left(T_{\mathrm{Top}}\right)-J_{e}\left(T_{\mathrm{Bot}}\right)$. One then finds in the ballistic case and in the absence of phonons for the small temperature difference $T_{\text {top }}-T_{\text {bot }}$

$$
J_{T}=\frac{d J_{e}(T)}{d T}\left(T_{\text {top }}-T_{\text {bot }}\right)
$$

and, therefore,

$$
\kappa_{x y}=\rho_{y x}^{\mathrm{th}-1}=\frac{d J_{e}(T)}{d T}, \quad \kappa_{x x}=\rho_{x x}^{\mathrm{th}}=0 .
$$

Here, the thermal resistivity tensor is defined as the inverse of the thermal conductivity tensor, $\hat{\rho}^{\text {th }}=\hat{\kappa}^{-1}$. To simplify notations, we assume in the following a system with $90^{\circ}$ rotation invariance such that $\kappa_{x x}=\kappa_{y y}$ and $\kappa_{x y}=-\kappa_{y x}$. In Eq. (2), we used that there is no temperature drop parallel to the direction of the edge channels, implying that $\rho_{x x}^{\text {th }}=0$ and therefore, also, $\kappa_{x x}=\left\{\rho_{x x}^{\text {th }} /\left[\left(\rho_{x x}^{\text {th }}\right)^{2}+\left(\rho_{x y}^{\text {th }}\right)^{2}\right]\right\}=0$.

The formula Eq. (2) is valid for a $2 \mathrm{D}$ system. Its generalization to three dimensions is obtained by dividing the result by the thickness $L_{z}$ of the system:

$$
\kappa_{x y}^{3 \mathrm{D}}=\frac{1}{L_{z}} \frac{d J_{e}(T)}{d T} .
$$

It is instructive to calculate the thermal derivative of the heat current for noninteracting electrons. For a single chiral fermionic channel with arbitrary dispersion $\epsilon_{k_{x}}$, one finds

$$
\frac{d J_{e}(T)}{d T}=\int \epsilon_{k_{x}} \frac{d f\left(\epsilon_{k}\right)}{d T} \frac{d \epsilon_{k_{x}}}{d \hbar k_{x}} \frac{d k_{x}}{2 \pi}=-\int_{\epsilon_{\min }}^{\epsilon_{\max }} \frac{\epsilon^{2} f^{\prime}(\epsilon)}{2 \pi \hbar k_{B} T} d \epsilon .
$$

The remaining integral can be done analytically, and one obtains the textbook result [30]

$$
\frac{1}{T} \frac{d J_{e}(T)}{d T}=\left(c_{R}-c_{L}\right) \frac{\pi k_{B}^{2}}{6 \hbar}+O\left(\exp \left[-\frac{\epsilon_{\min / \max }}{k_{B} T}\right]\right) .
$$

We have added the prefactor $c_{R}-c_{L}$, which for the single chiral fermionic channel considered above is equal to 1 . In general, it encodes the difference of the conformal charges of chiral conformal field theories describing the low-energy properties of the edge theory. It is important to note that correction to the quantized result Eq. (5) is exponentially suppressed when the temperature is smaller than the bulk gap, which enters as the cutoff $\epsilon_{\min / \max }$ of the edge theory.
The quantization with exponential precision holds even when the edge theory is interacting and disordered, which we will prove explicitly below; see Sec. III. For a single chiral Majorana channel, one obtains half of the result of the fermionic channel, $c_{R}=1 / 2, c_{L}=0$, resulting in

$$
\frac{\kappa_{x y}}{T}=\frac{1}{2} \frac{\pi k_{B}^{2}}{6 \hbar}
$$

with exponential precision in the absence of phonons.

In a layered three-dimensional system (e.g., a stack of weakly coupled Kitaev models in a perpendicular magnetic field) the number of edge channels $c_{R}-c_{L}$ is proportional to the number of layers $N_{L}$, resulting in a quantization of $d_{z} \kappa_{x y}^{3 \mathrm{D}}$, where $d_{z}=L_{z} / N_{L}$ is the distance of layers; see Eq. (3).

The quantization of $(1 / T)\left\{\left[d J_{e}(T)\right] /(d T)\right\}$ and the corresponding quantization of $\kappa_{x y} / T$ for a system with a bulk gap strongly suggests a topological origin for this effect. As discussed in the Introduction, it has been shown that this is indeed the case. The topology of the bulk theory is directly connected to a gravitational bulk and corresponding edge anomaly, which explains the robustness of the result for systems with a bulk gap (i.e., without phonons), and the fact that corrections are exponentially small in the ratio of a bulk gap and temperature.

\section{B. Nonquantized ballistic Hall effect in the presence of phonons}

We now consider a system with (acoustic) phonons, where, however, the size of the sample $L$ is much smaller than a characteristic scattering length of chiral edge excitations and phonons, $\ell_{e}^{\mathrm{ph}} \gg L$. Note that the meanfree path of phonons grows rapidly with decreasing temperature and, therefore, the ballistic heat transport by phonons, i.e., energy transport limited only by surface scattering of phonons, is routinely observed in insulators at very low temperatures, even for macroscopically large samples [45]. In Fig. 1(b), we have sketched a situation where phonons are present but do not couple to the edge mode. The sketch assumes that the phonons are completely ballistic so that there is no temperature gradient inside the sample.

In the ballistic limit, there are two independent channels to transport energy: the chiral edge mode and the bulk phonons. Correspondingly, the total heat current splits into an edge and a phonon contribution,

$$
J_{T}=J_{e}+J_{\mathrm{ph}}=\left(\frac{d J_{e}(T)}{d T}+G_{\mathrm{ph}}^{\mathrm{th}}\right)\left(T_{\mathrm{top}}-T_{\mathrm{bot}}\right),
$$

where $G_{\mathrm{ph}}^{\text {th }}$ is the ballistic conductance of the phonon system, which depends in a nonuniversal way on the properties of the contact. 
In this situation, obviously, no quantization of the thermal Hall effect can be expected. The first problem is that the phonons and the edge mode are not in thermal equilibrium, a local temperature of the full system cannot be defined, and thermometers attached to the same position will give different readings depending on whether they couple more to the phonons or to the edge mode. Two thermal contacts in the transverse direction will, therefore, measure a temperature difference $\Delta T_{e x}=\alpha\left(T_{\text {top }}-T_{\text {bot }}\right)$, where $0 \leq \alpha \leq 1$ is a nonuniversal factor that depends on the precise location and coupling of the thermal contact. Deep in the ballistic regime, the longitudinal energy gradient vanishes, $\rho_{x x}^{\text {th }}=0$. One then finds, in the ballistic regime, that

$$
\kappa_{x y}=\frac{\rho_{x y}^{\mathrm{th}}}{\left(\rho_{x x}^{\mathrm{th}}\right)^{2}+\left(\rho_{x y}^{\mathrm{th}}\right)^{2}}=\frac{J_{T}}{\Delta T_{e x}}=\frac{1}{\alpha}\left(\frac{d J_{e}(T)}{d T}+G_{\mathrm{ph}}^{\mathrm{th}}\right),
$$

which is nonuniversal and depends on the details of the contacts injecting the energy and used to measure the temperature drop. Depending on the contacts, it is possible to obtain values of $\kappa_{x y}$ that can be much larger or much smaller than the quantized value. As discussed in the Introduction, a recent experiment [36,37] managed to measure a quantized thermal Hall effect of an electronic fractional Hall effect by carefully designing a temperature probe (noise spectroscopy of electrons) with $\alpha \approx 1$ and by working in a regime where $\left\{\left[d J_{e}(T)\right] /(d T)\right\} \gg G_{\mathrm{ph}}^{\mathrm{th}}$.

For generic thermal contacts, however, we conclude that, in the ballistic limit, phonons destroy any quantization of thermal Hall effects.

\section{Approximately quantized Hall effect due to phonon coupling}

Finally, we consider a system in the thermodynamic limit where the scattering length of phonons with the edge mode and the phonon-phonon scattering length are much smaller than the system size, $\ell_{\mathrm{ph}}^{e}, \ell_{\mathrm{ph}}^{\mathrm{ph}} \ll L$. This is the relevant limit for practically all bulk experiments (with the exception of very low temperatures when phonon transport can become ballistic, as discussed above). In this limit, local temperatures and, therefore, also thermal resistivities are well defined. To avoid spurious effects of contacts, the standard procedure to measure the thermal resistivity tensor is to heat the system on one side (e.g., electrically) with a known power. Therefore, the total steady-state heat current $J_{T}$ is known. Thermometers aligned parallel and perpendicular to $J_{T}$ are then used to measure the temperature gradients in parallel and perpendicular directions, which allows us to deduce the thermal resistivity tensor and, by inversion, the thermal conductivity tensor. The main experimental error in such a setup is usually that the position of the thermometers may not be known with sufficient precision.
The coupling to phonons implies that ballistic edge channels do not exist any more. Energy can and will leak out of the edge channels into the bulk. The edge properties are, therefore, fundamentally different compared to the ballistic case without phonons. Also, $\kappa_{x x}$ arising from bulk phonons will be finite and typically much larger than $\kappa_{x y}$.

Nevertheless, there is a convincing line of argument that suggests that $\kappa_{x y}$ (but not $\rho_{x y}^{\text {th }}$ ) should remain approximately quantized. Ultimately, we expect that the conductivity tensor is a bulk quantity that can be calculated from a Drude formula for the total heat current density $\boldsymbol{j}_{H}$ arising from three contributions, $\boldsymbol{j}_{H}=\boldsymbol{j}_{s}+\boldsymbol{j}_{\mathrm{ph}}+\boldsymbol{j}_{s, p h}$ : the heat current of the (gapped) bulk theory of the electronic or spin degrees of freedom $\boldsymbol{j}_{s}$, the heat current of the phonons $\boldsymbol{j}_{\mathrm{ph}}$, and the heat current arising from the coupling between the two; see Sec. IV. If one assumes that the phonons and the mixed terms give only small contributions, it is plausible that essentially only the quantized response of the gapped topological phase is measured.

A main goal of our paper is to consider this question not from the viewpoint of the bulk theory but from the viewpoint of an edge theory coupled to phonons. We exploit the fact that all gapped degrees of freedom can be integrated out and it has to be possible to reformulate the theory only in terms of the edge channel and bulk phonons without using properties of gapped bulk modes. Our goal is to clarify how the coupling to phonons can restore an approximate quantization of $\kappa_{x y}$ in a theory where the edge channels are not ballistic and where $\kappa_{x x}$ is large.

The starting point of our analysis is the continuity equation for the thermal edge current. We consider a strip of width $d$ along the edge of the sample and define the total energy density $E_{d}(x)=\int_{0}^{d} d y e(x, y)$ as a function of the coordinate $x$ parallel to the edge by integrating over the strip. Similarly, the heat current $J_{d}(x)=\int_{0}^{d} d y j_{H}^{x}(x, y)$ is defined as an integral of the total heat current density in the $x$ direction. Note that both $e$ and $j_{H}^{x}$ include contributions from the edge modes, phonons, and their interactions. We use a two-dimensional notation here but all arguments can easily be generalized to a three-dimensional system where the edge is a planar system.

In this case, the continuity equation reads

$$
\partial_{t} E_{d}+\nabla_{x} J_{d}=j_{\perp, d}^{\mathrm{ph}} .
$$

Here, $j_{\perp, d}^{\mathrm{ph}}(x)$ is the heat current in the direction perpendicular to the edge at coordinates $(x, d)$; see Fig. 1(d). We choose $d$ to be sufficiently far away from the edge so that $j_{\perp, d}^{\mathrm{ph}}$ is purely carried by bulk phonons with no contribution arising from edge states or the coupling of the edge states to the phonons.

We will now use that, in the linear response limit, there is a local thermal equilibrium of the edge mode and the bulk phonons due to their coupling. Furthermore, due to the 
presence of the chiral edge state, $J_{d}=J_{d}(T)$ is finite at finite temperatures. In the steady state, we have $\partial_{t} E_{d}=0$; therefore, the edges pump or absorb energy current into the bulk of the system:

$$
j_{\perp, d}^{\mathrm{ph}}=\nabla_{x} J_{d}=\frac{d J_{d}}{d T} \nabla_{x} T .
$$

The first equality in Eq. (10) follows from the continuity equation at the steady state. For the second equality, we expand $J_{d}$ in powers of $\nabla T, \quad J_{d}(x)=J_{d}[T(x)]+$ $\kappa_{e} \nabla_{x} T(x)+O\left[(\nabla T)^{2}\right]$, where the first term is the heat current in thermal equilibrium. The contribution from the second term to Eq. (10), $\nabla_{x}\left(\kappa_{e} \nabla T\right)=\left[\left(d \kappa_{e}\right) /(d T)\right]\left(\nabla_{x} T\right)^{2}+$ $\kappa_{e} \nabla_{x}^{2} T$, vanishes in the linear response, large $L$ limit. Thus, only the first one, $\nabla_{x} J_{d}[T(x)]=\left[\left(d J_{d}\right) /(d T)\right] \nabla_{x} T$, needs to be kept.

Equation (10) is the direct manifestation of a thermal edge anomaly: a gradient in temperatures pumps energy into the edge of the system, which is then transported away by phonons. We will show in Sec. III that $\left[\left(d J_{d}\right) /(d T)\right]$ can be identified with the edge anomaly $\left[\left(d J_{e}\right) /(d T)\right]$ from Eq. (5) in the absence of phonons.

At the next step, we investigate what pattern of thermal gradient emerges due to the injection of the heat from the edge channel into the phonon system. The total heat current in the $x$ direction has two contributions, one from the bulk phonons and a further contribution arising from the temperature difference $L_{y} \nabla_{y} T$ of the edge modes (as discussed in Sec. II A):

$$
\frac{J_{T}}{L_{y}}=j_{\|}^{\mathrm{ph}}+\frac{d J_{d}}{d T} \nabla_{y} T
$$

The temperature gradients measured experimentally are obtained from the energy current densities $j_{\|}^{\mathrm{ph}}$ and $j_{\perp}^{\mathrm{ph}}$ and the bulk conductivity matrix of the phonons,

$$
\hat{\kappa}^{\mathrm{ph}} \cdot\left(\begin{array}{c}
\nabla_{x} T \\
\nabla_{y} T
\end{array}\right)=\left(\begin{array}{c}
j_{\|}^{\mathrm{ph}} \\
j_{\perp}^{\mathrm{ph}}
\end{array}\right)=\left(\begin{array}{c}
\frac{J_{T}}{L_{y}}-\frac{d J_{d}}{d T} \nabla_{y} T \\
\frac{d J_{d}}{d T} \nabla_{x} T
\end{array}\right),
$$

where we used Eqs. (10) and (11). The interpretation of the experiment assumes that there is no current flowing in the perpendicular direction and that the current density in the parallel direction is given by $J_{T} / L_{y}$. Therefore, $\nabla_{x} T=$ $\rho_{x x}^{\text {th }} J_{T} / L_{y}$ and $\nabla_{y} T=\rho_{x y}^{\text {th }} J_{T} / L_{y}$. By inverting the $\hat{\rho}^{\text {ph }}$ matrix, we find

$$
\kappa_{x y}=\frac{d J_{d}}{d T}+\kappa_{x y}^{\mathrm{ph}} \quad \kappa_{x x}=\kappa_{x x}^{\mathrm{ph}} .
$$

This result, derived from the edge theory, has a straightforward interpretation: The heat conductivity of the lowenergy bulk phonons has to be added to the conductivity arising from the edge channel-as expected from the bulkboundary correspondence.

This simple addition rule-while highly plausible - is a nontrivial result because usually (heat) conductivities are not additive. One can write the total heat current $\boldsymbol{j}=$ $\boldsymbol{j}_{\mathrm{ph}}+\boldsymbol{j}_{s}+\boldsymbol{j}_{s, \mathrm{ph}}$ as a sum of contributions arising from phonons, spins, and their interaction, and then apply the appropriate Kubo formula in order to calculate the thermal conductivity. In that case, the Kubo formula will contain nine different terms, which describe, for example, how the phonon heat currents influence spin-heat currents and vice versa. Ultimately, the bulk gap of the spin system is the reason why a simple addition law is valid.

As the phonon Hall effect $\kappa_{x y}^{\mathrm{ph}}$ is much smaller than the contribution $\left[\left(d J_{d}\right) /(d T)\right]$ from the edge anomaly (see Sec. IV), one can expect that the measured thermal Hall effect is dominated by the edge anomaly and, thus (taking the arguments of the next section into account), approximately quantized. We would like to repeat that the coupling of edge modes and phonons was absolutely essential to derive this result; see Sec. II B. The phonon coupling enforces the temperature gradient that gives rise to the pumping of energy from the edge into the bulk of the system.

According to Eq. (10), there is a dissipative phonon heat current (i.e., an energy current in the direction of a temperature gradient) flowing in the perpendicular direction. For energy-conserving boundary conditions, such currents usually (i.e., in the absence of edge anomalies) do not occur in the transverse direction. Here, however, it is important to stress that the entropy production proportional to $j_{\perp}^{\mathrm{ph}} \nabla_{y}(1 / T)$ is exactly canceled by the reduction of the current in the parallel direction arising from the edge mode, which contributes proportionally to $-\left[\left(d J_{d}\right) /(d T)\right] \nabla_{y} T \nabla_{x}(1 / T)$ to the entropy production.

\section{EDGE ANOMALY IN THE PRESENCE OF PHONON COUPLING}

Next, we investigate how the equilibrium edge current in the absence of phonons $J_{e}(T)$ is related to the equilibrium edge current in the presence of phonons $J_{d}(T)$. As the coupling of the edge modes to the phonons immediately destroys the ballistic transport of nonequilibrium edge currents by allowing leakage into the bulk, one may worry that it also modifies the equilibrium edge current. This is directly related to the question of whether the presence of gapless phonons modifies the gravitational anomaly in the bulk and on the edge. We will first discuss a nonperturbative argument and then check some of its assumptions by a perturbative calculation in the Appendix.

Our goal will be to investigate how $J_{d}(T)=\left\langle\hat{J}_{d}\right\rangle$ changes when the edge Hamiltonian is changed. We describe the change by a single parameter $\lambda_{e}$. Changing $\lambda_{e}$, e.g., from 0 to 1 may change the band curvature, induce a coupling among edge channels, or switch on interactions 
and disorder along the edge (to simplify the calculation we do, however, assume translational invariance below). Most importantly, we assume that, for $\lambda_{e}=0$, there is no coupling to the phonons, while, for $\lambda_{e}>0$, the coupling to acoustic bulk phonons is activated. Denoting the equilibrium density matrix by $\rho$, two contributions may affect $J_{d}$ :

$$
\frac{d}{d \lambda_{e}} J_{d}(T)=\operatorname{Tr}\left[\frac{\partial \hat{J}_{d}}{\partial \lambda_{e}} \rho\right]+\operatorname{Tr}\left[\hat{J}_{d} \frac{\partial \rho}{\partial \lambda_{e}}\right] .
$$

The second term can be identified with a standard retarded correlation function, well known from linear response theory,

$$
\begin{aligned}
\operatorname{Tr}\left[\hat{J}_{d} \frac{\partial \rho}{\partial \lambda_{e}}\right]= & \lim _{q_{x} \rightarrow 0} \lim _{\omega \rightarrow 0} \chi_{\lambda_{e}}\left(q_{x}, \omega\right) \\
\chi_{\lambda_{e}}\left(q_{x}, \omega\right)= & -\frac{i}{\hbar L_{x}} \int_{0}^{\infty} d t e^{i(\omega+i \epsilon) t} \\
& \times\left\langle\left[j_{d}\left(q_{x}, t\right), \frac{\partial e_{d}\left(-q_{x}, 0\right)}{\partial \lambda_{e}}\right]\right\rangle,
\end{aligned}
$$

with $L_{x}$ the length of the system and an infinitesimal $\epsilon>0$. As we calculate the change of a thermal equilibrium expectation value, the susceptibility has to be evaluated in the limit where one takes first $\omega \rightarrow 0$ and then $q_{x} \rightarrow 0$ (in contrast, dc conductivities are calculated using the opposite order of limits). Note that we introduced the Fourier transformation to momentum space (but not frequency space) for the energy current density and the $\lambda_{e}$ derivative of the energy density evaluated in a strip of width $d$ along the edge. Using the Fourier-transformed version of Eq. (9), $i q_{x} j_{d}\left(q_{x}, t\right)=-\left\{\left[d e_{d}\left(q_{x}, t\right)\right] /(d t)\right\}+j_{\mathrm{ph}, d}^{\perp}\left(q_{x}, t\right)$, we split $\chi_{\lambda_{e}}$ into two terms, $\chi_{\lambda_{e}}=\chi_{\lambda_{e}}^{\|}+\chi_{\lambda_{e}}^{\perp}$, where the first one is given by

$$
\begin{aligned}
\chi_{\lambda_{e}}^{\|}\left(q_{x}, 0\right) & =\frac{1}{\hbar L_{x} q_{x}} \int_{0}^{\infty} d t e^{-\epsilon t}\left\langle\left[\frac{d e_{d}\left(q_{x}, t\right)}{d t}, \frac{\partial e_{d}\left(-q_{x}, 0\right)}{\partial \lambda_{e}}\right]\right\rangle \\
& =-\frac{1}{\hbar L_{x} q_{x}}\left\langle\left[e_{d}\left(q_{x}, 0\right), \frac{\partial e_{d}\left(-q_{x}, 0\right)}{\partial \lambda_{e}}\right]\right\rangle \\
& =-\int d x d x^{\prime} \frac{e^{i q_{x}\left(x-x^{\prime}\right)}}{\hbar L_{x} q_{x}}\left\langle\left[e_{d}(x), \frac{\partial e_{d}\left(x^{\prime}\right)}{\partial \lambda_{e}}\right]\right\rangle,
\end{aligned}
$$

where we use the Fourier transformation back to position space. In the limit $q_{x} \rightarrow 0, \chi_{\lambda_{e}}^{\|}$evaluates to

$$
\begin{aligned}
\chi_{\lambda_{e}}^{\|}\left(q_{x} \rightarrow 0,0\right)= & -\frac{1}{\hbar L_{x}} \int d x d x^{\prime} i\left\langle\left[x e_{d}(x), \frac{\partial e_{d}\left(x^{\prime}\right)}{\partial \lambda_{e}}\right]\right. \\
& \left.+\left[x \frac{\partial e_{d}(x)}{\partial \lambda_{e}}, e_{d}\left(x^{\prime}\right)\right]\right\rangle=-\left\langle\frac{\partial J_{d}}{\partial \lambda_{e}}\right\rangle,
\end{aligned}
$$

where we used that the heat current along the edge can be written (using the continuity equation) as $L_{x} J_{d}=\int d x d x^{\prime} i\left\langle\left[x e_{d}(x), e_{d}\left(x^{\prime}\right)\right]\right\rangle$. The contribution from $\chi_{\lambda_{e}}^{\|}$, therefore, cancels exactly the first term in Eq. (14), and the only remaining term is

$$
\begin{aligned}
\frac{d J_{d}}{d \lambda_{e}}= & \lim _{q_{x} \rightarrow 0} \lim _{\omega \rightarrow 0} \chi \chi_{\lambda_{e}}^{\perp}\left(q_{x}, \omega\right) \\
\chi \frac{\perp}{\lambda_{e}}\left(q_{x}, \omega\right)= & \frac{1}{L_{x} \hbar} \int_{0}^{\infty} d t \frac{e^{i(\omega+i \epsilon) t}}{q_{x}} \\
& \times\left\langle\left[j j_{\mathrm{ph}, d}^{\perp}\left(q_{x}, t\right), \frac{\partial e_{d}\left(-q_{x}, 0\right)}{\partial \lambda_{e}}\right]\right\rangle \\
= & 0 \quad \text { for } d \rightarrow \infty .
\end{aligned}
$$

We have checked in a perturbative calculation to order $\lambda_{e}{ }^{2}$ that $\lim _{q_{x} \rightarrow 0} \lim _{\omega \rightarrow 0} \chi_{\lambda_{e}}^{\perp}\left(q_{x}, \omega\right) \sim\left[\left(\lambda_{e}^{2}\right) / d^{2}\right]$ in two dimensions; see the Appendix. The fact that $\chi \frac{\perp}{\lambda_{e}}$ vanishes for large $d$ is plausible because, by definition, $\lambda_{e}$ affects only the region close to the edge, while $j_{\perp}$ is measured at a distance $d$ from the edge. Because of the gapless phonon modes, one obtains a power-law decay with $d$.

For sufficiently large $d$, we can, therefore, conclude that $d J_{d} / d \lambda_{e}=0$ : The coupling of the edge modes to the gapless phonon modes in the bulk has no effect on the edge anomaly in the thermodynamic limit; therefore, the first term in Eq. (13) remains quantized.

\section{CONTRIBUTION OF BULK PHONONS TO THERMAL HALL CONDUCTIVITY}

In the previous section, we have shown that the edge contribution $d J_{d} / d T$ to the thermal Hall response, Eq. (13), is not affected by the coupling to phonons. To complete our discussion, we have to estimate the contribution $\kappa_{x y}^{\mathrm{ph}}$ of the bulk phonons to the thermal Hall effect. We ignore a tiny contribution arising for the direct coupling of the ions to an external magnetic field and study instead a - much largerBerry phase correction (an "anomalous" thermal Hall effect) arising due to the coupling of the phonons to the gapped bulk modes of the magnetic electronic or spin system.

For definiteness, we consider 2D phonons in a 2D Kitaev honeycomb model, but we expect that the main qualitative features of our result will be independent of the concrete model and generic for a large class of systems showing an approximately quantized thermal Hall effect. We will derive an effective low-energy theory for the phonons that takes into account the coupling to the Majorana degrees of freedom, and then we will calculate the thermal Hall conductivity of this purely phononic theory.

Our starting point is the effective low-energy Hamiltonian $H=H_{K}+H_{p}+H_{K p}$, consisting of the standard 2D Kitaev model [4] $H_{K}$, in the presence of an external magnetic field, the phonon Hamiltonian $H_{p}$, and the coupling of phonons to the Majorana spins. We assume that the coupling arises from the fact that the Kitaev coupling $J$ depends on the distance 
between atoms. The phonon-Majorana coupling constant is, therefore, given by $\lambda=[(d J) /(d r)]$, and we obtain

$$
\mathcal{H}_{I}=-\lambda \sum_{\mathbf{n}, j} \hat{\mathbf{M}}_{j} \cdot\left(\mathbf{u}_{\mathbf{n}}-\mathbf{u}_{\mathbf{n}+\mathbf{M}_{j}}\right) i b_{\mathbf{n}} a_{\mathbf{n}+\mathbf{M}_{j}}
$$

where $\hat{\boldsymbol{M}}_{j}(j=1,2,3)$ are normalized vectors connecting neighboring sites; $a$ and $b$ are the Majorana fermions on the different sublattices; and $\boldsymbol{u}_{n}$ is the displacement of the lattice atom at site $n$. In order to derive an effective low-energy theory, we expand about the Dirac points of the Kitaev model and consider only acoustical phonon modes, where we get

$\left.\mathcal{H}=\mathcal{H}_{p}+\hbar v_{M} \int d^{2} r \psi^{\dagger}([-i \nabla-\boldsymbol{A})] \cdot \boldsymbol{\sigma}+b \sigma_{z}\right) \psi$,

where $\psi$ is a two-component fermionic spinor (arising from combining two Majorana nodes to a single Dirac node); $b$ is a term due to the external magnetic field that drives the Majorana system into the quantum-Hall phase; $\mathcal{H}_{p}$ is the free phononic Hamiltonian; and $\mathbf{A}$ is the dynamic field phonon field that couples to the Majoranas, given by

$$
\mathbf{A}(\mathbf{r})=\frac{\lambda a}{\hbar v_{M}}\left(\begin{array}{l}
-\partial_{x} u_{x}(\mathbf{r})+\partial_{y} u_{y}(\mathbf{r}) \\
\partial_{y} u_{x}(\mathbf{r})+\partial_{x} u_{y}(\mathbf{r})
\end{array}\right),
$$

where $a$ is the distance of nearest neighbors and $\boldsymbol{A}$ acts like a vector potential, reflecting the well-known fact [46] that lattice distortions can act as a "synthetic" orbital magnetic field. In principle, there can also be further coupling terms, including a term $b \sigma_{z}\left(\partial_{x} u_{x}+\partial_{y} u_{y}\right)$, which will only renormalize the stiffness of the phonon lattice not contributing to $\kappa_{x y}^{\mathrm{ph}}$ to leading order.

Our next task is to integrate out the Majorana degrees of freedom and obtain an effective low-energy theory only for the phonons. Here, we can use that $\boldsymbol{A}$ couples to the fermionic current $\boldsymbol{j}$. It is, therefore, possible to compute the effective action of the phonons from the conductivity tensor $\sigma_{i j}(\omega)=\left[\left\langle j_{i} ; j_{j}\right\rangle /(i \omega)\right]$ of the fermionic quasiparticles obtained in the usual way from the current-current correlation function (while there is no charge conservation in the parent model, we can define a conductivity for the effective low-energy model). In the gapped quantum-Hall phase and for temperatures well below the gap, $\sigma_{x x}$ vanishes, but $\sigma_{x y}$ obtains the quantized value of half a conductance quantum:

$$
\sigma_{x y}=\lim _{\omega \rightarrow 0} \frac{\left\langle j_{x} ; j_{y}\right\rangle}{i \omega}=\operatorname{sign}(b) \frac{e^{2}}{4 \pi \hbar} .
$$

Integrating out the Majorana modes for small momenta $\boldsymbol{q}$ and frequencies $\omega$ results in a term in the Lagrangian of the form $\sum_{\boldsymbol{q}, \omega} A_{x}(-\omega,-\boldsymbol{q}) \sigma_{x y} i \omega A_{y}(\omega, \boldsymbol{q})$. Using $\hbar \partial_{t} A_{y} \approx$ $i\left[H_{p}^{0}, A_{y}\right]$, we obtain after some algebra the effective Hamiltonian for the acoustic phonons in 2D,

$$
\begin{aligned}
\mathcal{H}_{p} & =\mathcal{H}_{p}^{0}+\mathcal{H}_{p}^{H} \\
\mathcal{H}_{p} & =\int \frac{d^{2} k}{(2 \pi)^{2}} \frac{1}{2 \mu} \boldsymbol{\pi}_{\mathbf{k}} \boldsymbol{\pi}_{-\mathbf{k}}+\frac{\mu}{2} \mathbf{u}_{\mathbf{k}} \stackrel{\leftrightarrow}{M}_{\boldsymbol{k}} \mathbf{u}_{-\mathbf{k}} \\
\mathcal{H}_{p}^{H} & =\frac{\lambda^{2} a^{2} \operatorname{sign}(b)}{8 \pi \hbar v_{M}^{2} \mu} \int \frac{d^{2} k}{(2 \pi)^{2}}\left(k_{x}^{2}+k_{y}^{2}\right)\left(u_{\boldsymbol{k}}^{y} \pi_{-\mathbf{k}}^{x}-u_{\boldsymbol{k}}^{x} \pi_{-\mathbf{k}}^{y}\right),
\end{aligned}
$$

where $\pi$ is the momentum density of the ions, $\mu$ the $2 \mathrm{D}$ mass density of the solid, and $\overleftrightarrow{M}$ parametrizes the strain energy:

$$
\overleftrightarrow{M}(\mathbf{k})=\left[\begin{array}{cc}
u_{1}^{2}\left(k_{x}^{2}+k_{y}^{2}\right)+u_{2}^{2} k_{x}^{2} & u_{2}^{2} k_{x} k_{y} \\
u_{2}^{2} k_{x} k_{y} & u_{1}^{2}\left(k_{x}^{2}+k_{y}^{2}\right)+u_{2}^{2} k_{y}^{2}
\end{array}\right] .
$$

Because of the high symmetry of the honeycomb lattice, two parameters $u_{1}$ and $u_{2}$ are sufficient to parametrize the strain for $k \rightarrow 0$. Here, $u_{\perp}=u_{1}$ is the sound velocity of the transverse acoustic phonon, while the longitudinal acoustic phonon obtains the velocity $u_{\|}=\sqrt{u_{1}^{2}+u_{2}^{2}}$ for all directions of $\hat{k}$.

From the Hamiltonian of Eq. (21), one can derive the equations of motions for $\boldsymbol{u}_{k}$ and $\boldsymbol{\pi}_{k}$, which can be written into a matrix form $\dot{x}_{k}=-i \tilde{H}(\boldsymbol{k}) x_{k}$, with $x_{k}=$ $\left(\pi_{k}^{x}, \pi_{k}^{y}, u_{k}^{x}, u_{k}^{y}\right)^{T}$ and

$$
\tilde{H}(\boldsymbol{k})=\frac{i}{2 \mu}\left(\begin{array}{cc}
\Lambda(\boldsymbol{k}) & -\mu^{2} \stackrel{\leftrightarrow}{M}(\boldsymbol{k}) \\
I_{2} & \Lambda(\boldsymbol{k})
\end{array}\right)
$$

Here, $I_{2}$ is the $2 \times 2$ identity matrix and

$$
\Lambda(\boldsymbol{k})=\frac{\lambda^{2} a^{2} \operatorname{sign}(b)}{4 \pi \hbar v_{M}^{2}}\left(k_{x}^{2}+k_{y}^{2}\right)\left(\begin{array}{cc}
0 & 1 \\
-1 & 0
\end{array}\right) .
$$

This formulation allows us to calculate directly the phonon thermal Hall conductivity $\kappa_{x y}^{\mathrm{ph}}[41-43,47]$. To do so, we find the left and right eigenvectors of $\tilde{H}(\boldsymbol{k})$, labeling them $\xi_{k, \sigma}^{L / R}$, with the corresponding eigenvalues $\omega_{k, \sigma}$. Note that the four eigenvalues come in pairs $\omega_{k, \sigma}=-\omega_{-k,-\sigma}$, which are the frequencies of the corresponding creation and annihilation operators that diagonalize the Hamiltonian. The phonon Berry curvature is then given by [43]

$$
\Omega_{k, \sigma}^{z}=-\operatorname{Im}\left(\nabla_{k} \xi_{k, \sigma}^{L} \times \nabla_{k} \xi_{k, \sigma}^{R}\right)_{z}
$$

and $\kappa_{x y}^{\mathrm{ph}}$ is determined by $\Omega_{\boldsymbol{k}, \sigma}^{z}$ and $\omega_{\boldsymbol{k}, \sigma}$ by [43]

$$
\kappa_{x y}^{\mathrm{ph}}=-\frac{1}{\hbar T} \int_{0}^{\infty} d \epsilon \epsilon^{2} \sigma_{x y}^{\mathrm{ph}}(\epsilon) \frac{d n(\epsilon)}{d \epsilon},
$$

where 


$$
\sigma_{x y}^{\mathrm{ph}}(\epsilon)=-\sum_{\sigma>0} \int \frac{d^{2} k}{(2 \pi)^{2}} \Omega_{k, \sigma}^{z} \Theta\left(\epsilon-\hbar \omega_{k, \sigma}\right),
$$

with the sum going only over the bands with positive frequencies.

To order $\lambda^{2}$, the phonon frequencies $\omega_{k,(1,2)}=u_{(\|, \perp)} k+$ $O\left(\lambda^{4} k^{3}\right)$ are not affected by the Majorana-phonon coupling. The coupling does, however, induce the Berry curvature

$$
\begin{aligned}
& \Omega_{k, 1}^{z}=\frac{3 u_{\|}^{2}+u_{\perp}^{2}}{u_{\|}^{2}-u_{\perp}^{2}} \frac{\lambda^{2} a^{2} \operatorname{sign}(b)}{8 \pi \hbar v_{M}^{2} \mu} \frac{1}{u_{\|} k}+O\left(\lambda^{4}\right) \\
& \Omega_{k, 2}^{z}=-\frac{u_{\|}^{2}+3 u_{\perp}^{2}}{u_{\|}^{2}-u_{\perp}^{2}} \frac{\lambda^{2} a^{2} \operatorname{sign}(b)}{8 \pi \hbar v_{M}^{2} \mu} \frac{1}{u_{\perp} k}+O\left(\lambda^{4}\right)
\end{aligned}
$$

of the longitudinal and transversal phonons, respectively. Note that the Berry curvature displays a $1 / k$ divergence for $k \rightarrow 0$. This divergence arises because the Berry phase originates from a coupling of longitudinal and transversal phonons whose energy difference vanishes linear in $k$. The corresponding Berry flux is, however, finite. In contrast to, e.g., Berry curvatures characterizing Dirac nodes, it is not quantized and proportional to $\lambda^{2}$. The Berry phase nominally also diverges when the two sound velocities approach each other, $u_{\|} \rightarrow u_{\perp}$, or, equivalently, for $u_{2} \rightarrow 0$. Because of the opposite sign of the Berry curvature of the two band, this divergence does, however, cancel when the heat conductivity is calculated.

Using Eq. (26), we obtain, for two-dimensional phonons,

$$
\begin{aligned}
\frac{\kappa_{x y}^{\mathrm{ph}, 2 \mathrm{D}}}{T} & =\frac{k_{B}^{2}}{\hbar} \frac{u_{\|}^{2}+u_{\perp}^{2}}{u_{\|}^{2} u_{\perp}^{2}} \frac{3 \zeta(3) \lambda^{2} a^{2} \operatorname{sign}(b)}{8 \pi^{2} v_{M}^{2} \hbar \mu} k_{B} T+O\left(\lambda^{4}\right) \\
& \sim \frac{k_{B}^{2}}{\hbar} \operatorname{sign}(b)\left(\frac{a}{J} \frac{d J}{d r}\right)^{2} \frac{k_{B} T}{K_{2 \mathrm{D}} a^{2}},
\end{aligned}
$$

with $\zeta(x)$ the Riemann zeta function, $\zeta(3) \approx 1.2$. For the last line, we used $\hbar v_{M} \sim J a$ and identified $\mu u^{2}$ with the 2D bulk modulus $K_{2 \mathrm{D}}$ with units of force per length.

For three-dimensional phonons in a model of stacked Kitaev layers, the main difference is that the momentum integral in Eq. (27) becomes three dimensional. For comparison with the quantized heat conductivity, it is useful to consider the thermal conductivity per layer, obtained by multiplying the 3D heat conductivity with the layer distance $d_{z}$ :

$$
\frac{d_{z} k_{x y}^{\mathrm{ph}, 3 \mathrm{D}}}{T} \frac{k_{B}^{2}}{\hbar} \sim \operatorname{sign}(b)\left(\frac{a}{J} \frac{d J}{d r}\right)^{2} \frac{k_{B} T^{2}}{\Theta_{D} K a^{3}} .
$$

Here, $\Theta_{D}$ is the Debye temperature and $K$ is the 3D bulk modulus. Assuming, e.g., a Debye temperature of $200 \mathrm{~K}$, a bulk modulus $K$ of $100 \mathrm{GPa}$, a lattice constant of $2 \AA$, a temperature of $5 \mathrm{~K}$, and $[(d J) /(d r)] \sim 10(J / a)$, we obtain a phonon correction of the order of only $10^{-4}$.
The fact that phonon Berry curvatures give rise to $\kappa_{x y} \propto$ $T^{3}$ has been shown previously by Qin et al. [43]. In their case, however, the Berry curvature and, therefore, $\kappa_{x y}^{\mathrm{ph}}$ were proportional to the magnetization $M$ of the system. In contrast, we find an anomalous contribution that just depends on the sign of the magnetization (or, equivalently, of $b$ ), not on its absolute value. This can be traced back to the quantized $\sigma_{x y}$ of the bulk Majorana arising for an infinitesimal external magnetic field. We expect this feature to be independent of the precise microscopic model and an $M$ - or $B$-independent $T^{2}$ term in $\kappa_{x y} / T$ to be characteristic for a large class of chiral phases.

\section{DISCUSSION AND CONCLUSION}

In our paper, we put forward a straightforward interpretation of the approximately quantized Hall effect observed very recently in a chiral spin liquid state of $\alpha-\mathrm{RuCl}_{3}$ : In the presence of an energy current, the equilibration of the edge channel with the phonons induces a temperature gradient in the edge mode. This temperature gradient implies that there is a gradient in the steady-state heat current $J_{d}$ flowing at the edge. This gradient is given by $\left[\left(d J_{d}\right) /(d T)\right] \nabla T$, and its value is fixed by the gravitational anomaly of the edge mode. Energy conservation enforces that the energy current $j_{\perp, d}^{\mathrm{ph}}=\left[\left(d J_{d}\right) /(d T)\right] \nabla T$, Eq. (9), is injected into the phonon system in the perpendicular direction. This leads to an approximately quantized thermal Hall effect that can be measured by tracking temperature gradients in the phonon system only.

We have carefully checked that the amplitude of the edge anomaly (in an infinite system) is not affected by the coupling of the edge modes to the phonons. It remains topologically protected despite the gapless phonon modes in the bulk. The only correction arises from the thermal Hall effect of the bulk phonons. At least in a sufficiently clean system, we expect that the leading correction to the quantized thermal Hall effect arises from Berry phases imprinted on the acoustic phonons by the chiral spin liquid. In three dimensions, this leads to a correction to $\kappa_{x y} / T$ proportional to $T^{2}$ with a small prefactor, Eq. (31). Remarkably, this correction is, within our approximations, independent of the size of the external magnetic field $B$ or the magnetization of the system, but depends on the sign of $B$.

When discussing the temperature dependence of $\kappa_{x y}$, one has to take into account that, at the lowest temperatures, phonons become ballistic and stop to scatter from each other and the boundary. In this regime, the approximate quantization of $\kappa_{x y}$ is lost. Depending on the type of contacts used to inject the heat current and to measure the temperature, it can happen that one observes an apparent $\kappa_{x y} / T$, which is either much larger or much smaller than the quantized value; see Sec. II B. In Fig. 2, we show schematically the expected $T$ dependence of $\kappa_{x y} / T$. The condition for observing the 


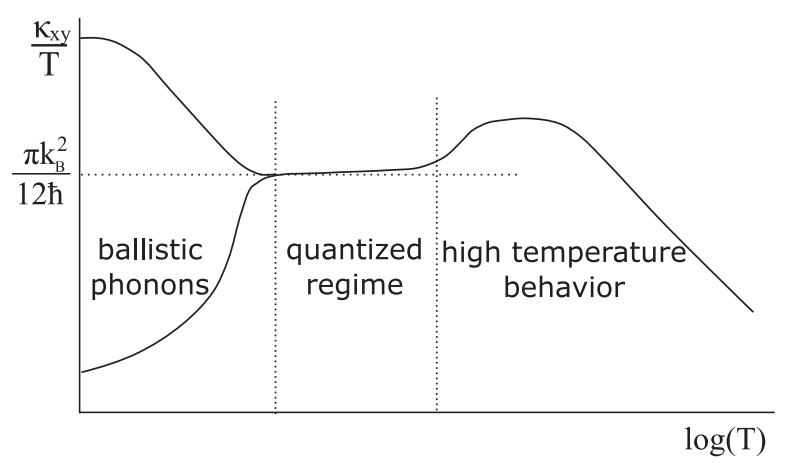

FIG. 2. Schematic plot of the $T$ dependence of $\kappa_{x y}$ per layer of a chiral (spin) liquid coupled to phonons. An approximately quantized Hall effect, $\left[\left(\kappa_{x y}\right) / T\right]=\left\{\left[\left(c_{r}-c_{l}\right) \pi k_{B}^{2}\right] /(6 \hbar)\right\}+$ $O\left(T^{2}\right)$, is observed for $T$ much smaller than the bulk gap $\Delta_{b}$ of the chiral liquid under the condition that the phonon-phonon and the phonon-edge scattering length be smaller than the sample size, $\ell_{\mathrm{ph}}^{\mathrm{ph}}, \ell_{\mathrm{ph}}^{e} \ll L$. The leading correction for clean systems arises from an intrinsic anomalous Hall effect of phonons, Eq. (31). At lower $T$, spins and phonons decouple (mean-free path larger than system size) and one obtains a nonuniversal result (see Sec. II B), which can be larger or smaller than the quantized value depending on contact properties. For $T \gg \Delta_{b}$, the thermal Hall effects of both the spins and the phonons are expected to decay rapidly with increasing temperatures.

quantized thermal Hall effect is that the temperature is well below the bulk gap $\Delta_{b}$ of the chiral liquid and that the scattering length of phonons with the edge and with themselves is smaller than the system size $L$. We expect that the range of approximate quantization becomes larger in samples with more disorder, as disorder will allow for a more efficient phonon-phonon and phonon-edge coupling. For $\alpha-\mathrm{RuCl}_{3}$, we expect that the phonon correction in the relevant temperature regime is tiny, on the permille level or below. In this material, a pronounced maximum in $\kappa_{x y}$, larger than the quantized value, is observed for $T \sim \Delta_{b}$. The origin of this peak is an interesting open question; a possible candidate is the coupling of chiral spin fluctuations to the phonons. For $T \gg \Delta_{b}$, one expects that both the phonon and spin contribution to $\kappa_{x y}$ drop rapidly.

The observation of a quantized thermal Hall effect is the most direct measurement proving the existence of a gapped chiral (spin) liquid. Phonons help rather than hinder the observation of this effect. It is an interesting question how this physics changes close to the quantum critical points where, e.g., as function of a magnetic field, the chiral liquid is destroyed and phonons scatter predominately from chiral critical fluctuations.

\section{ACKNOWLEDGMENTS}

We acknowledge useful discussion with Roser Valentí, Yuichi Kasahara, Paul van Loosdrecht, Yuval Oreg, and Ady Stern. Financial support by the DFG (project C02 of
CRC1238 and project A01 of CRC/TR183) is gratefully acknowledged.

Note added.-Recently, a preprint by $\mathrm{Ye}$ et al. was published on arXiv [48] that also emphasizes the importance of phonon coupling for the observation of a quantized thermal Hall effect by investigating the hydrodynamic equations in a finite system.

\section{APPENDIX: PERTURBATIVE ANALYSIS OF PHONON COUPLING TO THE CHIRAL MODE}

To corroborate the picture presented in Sec. III, we analyze perturbatively the effect of the phonon coupling on the heat current. We will present at the beginning a strictly 1D analysis and then, based on it, an analysis of the case where the 1D Majorana edge mode is coupled to 2D phonons, which will emphasize the role of $\chi_{\lambda}^{\perp}$ in Eq. (16) and its dependence on $d$, the distance from the edge.

\section{1. $1 \mathrm{D}$ case}

In 1D where $\chi_{\lambda}^{\perp}=0$, Eq. (16) predicts that turning on the phonon coupling has no effect on the heat current. We will first check this statement perturbatively. To this end, we will calculate explicitly the different contributions to the heat current quadratic in the phonon coupling and show that their integrals cancel.

We consider a general 1D interacting Hamiltonian consisting of chiral fermions coupled locally to a single phonon band, described by $\mathcal{H}=\int d x h(x)=\int d x\left[h_{0}(x)+\right.$ $\left.h_{\lambda}(x)\right]$, with

$$
\begin{aligned}
h_{0}(x)= & \int \frac{d k d k^{\prime}}{2 \pi} e^{-i\left(k-k^{\prime}\right) x} \epsilon_{\left[\left(k+k^{\prime}\right) / 2\right]} c_{k}^{\dagger} c_{k^{\prime}} \\
& +\int \frac{d q d q^{\prime}}{2 \pi} e^{-i\left(q-q^{\prime}\right) x} \omega_{\left[\left(q+q^{\prime}\right) / 2\right]} a_{q}^{\dagger} a_{q^{\prime}}, \\
h_{\lambda}(x)= & \int \frac{d k d k^{\prime} d q}{2 \pi} e^{-i\left(k-k^{\prime}-q\right) x} \lambda_{k, k^{\prime}}^{q} c_{k}^{\dagger} c_{k^{\prime}} a_{q}+\text { H.c. }
\end{aligned}
$$

We make no assumptions as to fermionic energy bands $\epsilon_{k}$, bosonic energy bands $\omega_{q}$, or the coupling coefficients $\lambda_{k, k^{\prime}}^{q}$.

We proceed to derive the heat current operator from the $1 \mathrm{D}$ continuity equation $\partial_{x} j(x)+\partial_{t} h(x)=0$ in momentum space

$$
j(K)=-\frac{1}{K} \int d x d x^{\prime} e^{-i K x}\left[h(x), h\left(x^{\prime}\right)\right],
$$

and the total heat current is then $J_{T}=\lim _{K \rightarrow 0} j(K) / L_{x}$, with $L_{x}=\delta_{k}(0)$ the size of the system. Carrying out the commutation relations and taking the limit $K \rightarrow 0$, we can divide the contributions to $j(K)$ into four parts, arising from the different components of the Hamiltonian, $\left\langle J_{T}\right\rangle=$ $J_{00}+J_{0 \lambda}+J_{\lambda 0}+J_{\lambda \lambda}$, with 


$$
\begin{aligned}
J_{00}= & \frac{1}{L_{x}} \int d k v_{k} \epsilon_{k}\left\langle c_{k}^{\dagger} c_{k}\right\rangle+\frac{1}{L_{x}} \int d q u_{q} \omega_{q}\left\langle a_{q}^{\dagger} a_{q}\right\rangle, \\
J_{0 \lambda}= & \frac{1}{L_{x}} \int d k d k^{\prime} d q\left\{\partial_{k}\left[\delta\left(k-k^{\prime}-q\right) \lambda_{k, k^{\prime}}^{q} \epsilon_{k}\right]+\partial_{k^{\prime}}\left[\delta\left(k-k^{\prime}-q\right) \lambda_{k, k^{\prime}}^{q} \epsilon_{k^{\prime}}\right]+\partial_{q}\left[\delta\left(k-k^{\prime}-q\right) \lambda_{k, k^{\prime}}^{q} \omega_{q}\right]\right. \\
& \left.-\delta\left(k-k^{\prime}-q\right) \lambda_{k, k^{\prime}}^{q} \frac{v_{k}+v_{k^{\prime}}+u_{q}}{2}\right\}\left\langle c_{k}^{\dagger} c_{k^{\prime}} a_{q}\right\rangle+\text { c.c., } \\
J_{\lambda 0}= & \frac{1}{L_{x}} \int d k d k^{\prime} d q \partial_{k} \delta\left(k-k^{\prime}-q\right) \lambda_{k, k^{\prime}}^{q}\left(\omega_{q}+\epsilon_{k^{\prime}}-\epsilon_{k}\right)\left\langle c_{k}^{\dagger} c_{k^{\prime}} a_{q}\right\rangle+\text { c.c., } \\
J_{\lambda \lambda}= & \frac{1}{L_{x}} \int d k d k^{\prime} d q d p \partial_{k} \delta\left(k-k^{\prime}-q\right) \lambda_{k, k^{\prime}}^{q} \lambda_{p+q, p}^{q *}\left\langle c_{k}^{\dagger} c_{k^{\prime}} c_{p}^{\dagger} c_{p+q}\right\rangle+\int d k d k^{\prime} d q d q^{\prime} \partial_{k} \delta\left(k-k^{\prime}-q\right) \lambda_{k, k^{\prime}}^{q} p_{p, k^{\prime}}^{p-k^{\prime} *}\left\langle c_{k}^{\dagger} c_{p} a_{p-k^{\prime}}^{\dagger} a_{q}\right\rangle \\
& -\int d k d k^{\prime} d q d q^{\prime} \partial_{k} \delta\left(k-k^{\prime}-q\right) \lambda_{k, k^{\prime}}^{q} \lambda_{k, p}^{k-p *}\left\langle c_{p}^{\dagger} c_{k^{\prime}} a_{k-p}^{\dagger} a_{q}\right\rangle+\text { c.c. }+\cdots,
\end{aligned}
$$

with $\hbar v_{k}=\partial_{k} \epsilon_{k}$ and $\hbar u_{q}=\partial_{q} \omega_{q}$, and the $\ldots$ in the expression for $J_{\lambda \lambda}$ stands for terms containing two bosonic annihilation or creation operators, which will not contribute to the current at order $\lambda^{2}$.

We now show that the leading corrections of order $\lambda^{2}$ cancel when summing all the contributions. We start by focusing on $J_{00}$ and $J_{0 \lambda}$, and we expand the expectation values to evaluate the leading contribution in $\lambda^{2}$, by calculating the diagrams in Fig. 3. This results in

$$
\begin{aligned}
& J_{00}= J_{00}^{0}-\frac{1}{L_{x}} \int d k d k^{\prime} d q \delta^{2}\left(k-k^{\prime}-q\right)\left|\lambda_{k, k^{\prime}}^{q}\right|^{2}\left[\frac{v_{k} \epsilon_{k}}{\beta^{2}} \sum_{n, m} g_{k}^{2}\left(i \omega_{n}\right) g_{k^{\prime}}\left(i \omega_{m}\right) d_{q}\left(i \omega_{n}-i \omega_{m}\right)\right. \\
&\left.+\frac{v_{k^{\prime}} \epsilon_{k^{\prime}}}{\beta^{2}} \sum_{n, m} g_{k}\left(i \omega_{n}\right) g_{k^{\prime}}^{2}\left(i \omega_{m}\right) d_{q}\left(i \omega_{n}-i \omega_{m}\right)+\frac{u_{q} \omega_{q}}{\beta^{2}} \sum_{n, m} g_{k}\left(i \omega_{n}\right) g_{k^{\prime}}\left(i \omega_{m}\right) d_{q}^{2}\left(i \omega_{n}-i \omega_{m}\right)\right]+O\left(\lambda^{4}\right), \\
& J_{0 \lambda}=\frac{2}{\hbar L_{x}} \operatorname{Re} \int d k d k^{\prime} d q\left\{\partial_{k}\left[\delta\left(k-k^{\prime}-q\right) \lambda_{k, k^{\prime}}^{q} \epsilon_{k}\right]+\partial_{k^{\prime}}\left[\delta\left(k-k^{\prime}-q\right) \lambda_{k, k^{\prime}}^{q} \epsilon_{k^{\prime}}\right]+\partial_{q}\left[\delta\left(k-k^{\prime}-q\right) \lambda_{k, k^{\prime}}^{q} \omega_{q}\right]\right. \\
&\left.-\delta\left(k-k^{\prime}-q\right) \lambda_{k, k^{\prime}}^{q} \hbar \frac{v_{k}+v_{k^{\prime}}+u_{q}}{2}\right\} \delta\left(k-k^{\prime}-q\right) \lambda_{k, k^{\prime}}^{q *} \frac{1}{\beta^{2}} \sum_{n, m} g_{k}\left(i \omega_{n}\right) g_{k^{\prime}}\left(i \omega_{m}\right) d_{q}\left(i \omega_{n}-i \omega_{m}\right)+O\left(\lambda^{4}\right),
\end{aligned}
$$

with $g_{k}\left(i \omega_{n}\right)=\left(i \omega_{n}-\epsilon_{k}\right)^{-1}, d_{q}\left(i \Omega_{n}\right)=\left(i \Omega_{n}-\omega_{q}\right)^{-1}$ the bare Matsubara Green functions of the fermions and the phonons, $\omega_{n}=(2 n+1) \pi / \beta\left(\Omega_{n}=2 n \pi / \beta\right)$ the Matsubara fermionic (bosonic) frequencies, and $\beta$ the inverse temperature. In the expression for $J_{00}$, the term $J_{00}^{0}$ stands for the noninteracting $\lambda^{0}$ contribution, and we have changed variables $k \leftrightarrow k^{\prime}$ to get the second term in the parentheses in the integrand, with the multiplier $v_{k^{\prime}} \varepsilon_{k^{\prime}}$. To see the cancellation between the $\lambda^{2}$ contribution, one first uses

$$
\operatorname{Re}\left\{\left[2 \partial_{k}\left(f_{k, k^{\prime}}^{q} \epsilon_{k}\right)-f_{k, k^{\prime}}^{q} \hbar v_{k}\right] f_{k, k^{\prime}}^{q *}\right\}=\partial_{k}\left[\left|f_{k, k^{\prime}}^{q}\right|^{2} \epsilon_{k}\right]
$$

with $f_{k, k^{\prime}}^{q}=\delta\left(k-k^{\prime}-q\right) \lambda_{k, k^{\prime}}^{q}$ to reorder terms in Eq. (A5). Then, using integration by parts, while noticing that $\partial_{k} g_{k}=-\hbar v_{k} g_{k}^{2}, \partial_{q} d_{q}=-\hbar u_{q} d_{q}^{2}$, we see that the terms in $J_{0 \lambda}$ attain the exact form of the terms in $J_{00}$, but with the opposite sign. Boundary terms are exponentially small in temperature and are neglected.
Next, we show that the leading contributions to $J_{\lambda \lambda}$ and to $J_{\lambda 0}$ cancel each other as well. Evaluating the expectation values to leading order, we get

$$
\begin{aligned}
J_{\lambda 0}= & \frac{1}{\hbar L_{x}} \int d k d k^{\prime} d q \partial_{k} \delta^{2}\left(k-k^{\prime}-q\right)\left|\lambda_{k, k^{\prime}}^{q}\right|^{2} \\
& \times\left[f\left(\epsilon_{k^{\prime}}\right)+n\left(-\omega_{q}\right)\right]\left[f\left(\epsilon_{k}\right)-f\left(\epsilon_{k^{\prime}}+\omega_{q}\right)\right]+O\left(\lambda^{4}\right), \\
J_{\lambda \lambda}= & \frac{1}{\hbar L_{x}} \int d k d k^{\prime} d q \partial_{k} \delta^{2}\left(k-k^{\prime}-q\right)\left|\lambda_{k, k^{\prime}}^{q}\right|^{2} \\
& \times\left\{f\left(\epsilon_{k}\right)\left[\left(1-f\left(\epsilon_{k^{\prime}}\right)\right]+\left[f\left(\epsilon_{k}\right)-f\left(\epsilon_{k^{\prime}}\right)\right] n\left(\omega_{q}\right)\right\}\right. \\
& +O\left(\lambda^{4}\right),
\end{aligned}
$$

where $f(\epsilon)$ and $n(\omega)$ are the Fermi-Dirac and BoseEinstein distributions, respectively. These contributions also sum to zero.

To conclude, we have shown that, in $1 \mathrm{D}$, the fermionphonon interaction does not affect the heat current to order 
(a)

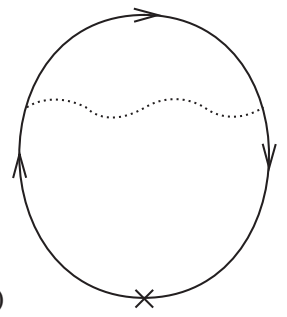

(b)

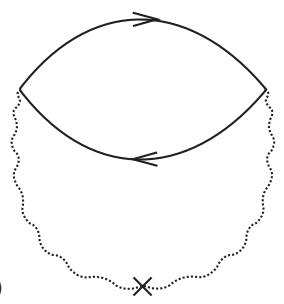

(c)

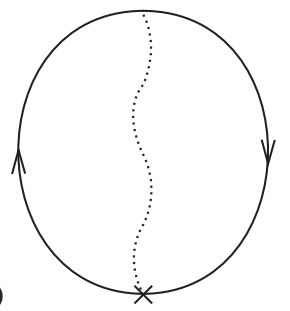

(d)

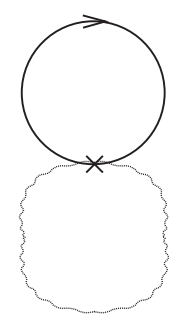

FIG. 3. Feynman diagrams used in calculating the expectation values of the heat current to leading order. Here, straight continuous lines are the fermion propagators and dotted wiggly lines are phonon propagators. The " $x$ " marks the space-time point at which the expectation value is evaluated. The different diagrams are (a) the $\lambda^{2}$ contribution to $\left\langle c_{k}^{\dagger} c_{k}\right\rangle$, (b) the $\lambda^{2}$ contribution to $\left\langle a_{\boldsymbol{q}}^{\dagger} a_{\boldsymbol{q}^{\prime}}\right\rangle$, (c) the $\lambda$ contribution to $\left\langle c_{k}^{\dagger} c_{k^{\prime}} a_{\boldsymbol{q}}\right\rangle$, and (d) the $\lambda^{0}$ contribution to $\left\langle c_{k}^{\dagger} c_{k^{\prime}} a_{q}^{\dagger} a_{q^{\prime}}\right\rangle$.

$\lambda$, a result consistent with the nonperturbative proof in the main text.

\section{2D case}

The generalization of the $1 \mathrm{D}$ case to a model that includes 2D phonons is straightforward. The local free phonon Hamiltonian now reads

$$
h_{0}^{\mathrm{ph}}(\boldsymbol{r})=\int \frac{d^{2} q d^{2} q^{\prime}}{(2 \pi)^{2}} e^{-i\left(\boldsymbol{q}-\boldsymbol{q}^{\prime}\right) \boldsymbol{r}} \omega_{\left[\left(\boldsymbol{q}+\boldsymbol{q}^{\prime}\right) / 2\right]} a_{\boldsymbol{q}}^{\dagger} a_{\boldsymbol{q}^{\prime}},
$$

and all integrals over phonon momenta $q$ in $h_{\lambda}$ are now two dimensional. Notice, however, that the coupling is still restricted to $y=0$ and the Majorana mode, as well as the coupling, is $1 \mathrm{D}$. While momentum is still conserved in the $x$-direction, the coupling to the edge mode at $y=0$ breaks momentum conservation in the $y$-direction. The formulation here is slightly different than in Sec. II C, as the phonons travel on both sides of the Majorana edge mode. This is more convenient for computational reasons, and we believe that it does not affect the main features of the heat current that we are interested in.

We are interested in the total heat current in the $x$-direction, going through a region of width $d$ in the $y$ direction $J_{d}=\int_{-\infty}^{\infty} d x \int_{-d / 2}^{d / 2} d y j_{x}(\boldsymbol{r})$. In the limit $d \rightarrow \infty$, the arguments presented in the 1D case hold, and we can again show that all contributions of order $\lambda^{2}$ vanish. For finite $d$, however, heat current can leak outside the region $d$ in the perpendicular direction. It will be carried only by phonons, and we can evaluate it as

$$
\Delta J_{x}^{\mathrm{ph}}(d)=J_{x}^{\mathrm{ph}, \mathrm{inf}}-\int d x \int_{-d / 2}^{d / 2} d y j^{\mathrm{ph}}(\boldsymbol{r}),
$$

where $j^{\text {ph }}$ is the heat current carried by the phonons, and $J_{x}^{\mathrm{ph}, \text { inf }}=\int d^{2} r j_{x}^{\mathrm{ph}}(\boldsymbol{r})$.

We write $\int d x j_{x}^{\mathrm{ph}}(\boldsymbol{r})$ to leading order in the Majoranaphonon interaction explicitly as

$$
\begin{aligned}
\int d x j_{x}^{\mathrm{ph}}(\boldsymbol{r})= & \int \frac{d k d k^{\prime} d q_{y} d q_{y}^{\prime}}{2 \pi} e^{-i\left(q_{y}-q_{y}^{\prime}\right) y} u_{\left(k-k^{\prime}, q_{y}\right)}^{x} \omega_{\left(k-k^{\prime}, q_{y}\right)} \lambda_{k, k^{\prime}}^{\left(k-k^{\prime}, q_{y}\right)} \lambda_{k^{\prime}, k}^{\left(k-k^{\prime}, q_{y}^{\prime}\right) *}\left[f\left(\epsilon_{k^{\prime}}\right)-f(\epsilon k)\right] \\
& \times \frac{\left(\omega_{\left(k-k^{\prime}, q_{y}^{\prime}\right)}-\epsilon_{k}+\epsilon_{k^{\prime}}\right) n\left(\omega_{\left(k-k^{\prime}, q_{y}\right)}\right)-\left(\omega_{\left(k-k^{\prime}, q_{y}\right)}-\epsilon_{k}+\epsilon_{k^{\prime}}\right) n\left(\omega_{\left(k-k^{\prime}, q_{y}^{\prime}\right)}\right)+\left(\omega_{\left(k-k^{\prime}, q_{y}\right)}-\omega_{\left(k-k^{\prime}, q_{y}^{\prime}\right)}\right) n\left(\epsilon_{k}-\epsilon_{k^{\prime}}\right)}{\left(\omega_{\left(k-k^{\prime}, q_{y}\right)}-\omega_{\left(k-k^{\prime}, q_{y}^{\prime}\right)}\right)\left(\omega_{\left(k-k^{\prime}, q_{y}\right)}-\epsilon_{k}+\epsilon_{k^{\prime}}\right)\left(\omega_{\left(k-k^{\prime}, q_{y}\right)}-\epsilon_{k}+\epsilon_{k^{\prime}}\right)} .
\end{aligned}
$$

Note that the heat current is proportional to $\lambda^{2}$, as the bare heat current of the phonons is zero due to the fact that the energy band of the phonons is an even function of the momentum $\omega_{q}=\omega_{-q}$, which makes $\boldsymbol{u}_{q} \omega_{q} n\left(\omega_{q}\right)$ an odd function whose integral vanishes.

To evaluate $\Delta J_{x}^{\mathrm{ph}}(d)$ numerically, we consider a specific model, namely, that of a Majorana edge state with dispersion $\epsilon_{k}=v_{M} k$ coupled locally to a single band of acoustic 2D phonons with dispersion $\omega_{q}=u q$. For coupling to electrons, the minimal coupling is to their density $h_{\lambda}(x)=$ $\lambda \partial_{x} \phi(x) \psi^{\dagger}(x) \psi(x)$, where $\phi(x)$ is the phononic field. However, such a coupling is not possible for a Majorana edge, as $\psi^{\dagger}(x) \psi(x)=\psi^{2}(x) \sim 1$, and we must consider coupling of the form $h_{\lambda}(x)=\lambda \partial_{x} \phi(x) \psi^{\dagger}(x)\left(-i \partial_{x}\right) \psi(x)$. This results in $\lambda_{k, k^{\prime}}^{q}=\lambda_{0} \hbar v_{M}(k+p) q_{x} / \sqrt{\omega_{q}} \exp \left(-\omega_{q} / \omega_{D}\right)$, where $\lambda_{0}$ is a coupling constant with dimensions of the square root of energy times area, and we introduced the Debye energy $\omega_{D}$ via a soft cutoff on the coupling. Finally, for computational convenience, we slightly change the formula for $\Delta J_{x}^{\mathrm{ph}}(d)$ by replacing the hard cutoff at $\pm d / 2$ with a soft Gaussian of width $d$

$$
\Delta J_{x}^{\mathrm{ph}}(d) \simeq J_{x}^{\mathrm{ph}, \mathrm{inf}}-\frac{1}{\sqrt{\pi}} \int d^{2} r e^{-(y / d)^{2}} j^{\mathrm{ph}}(\boldsymbol{r}),
$$



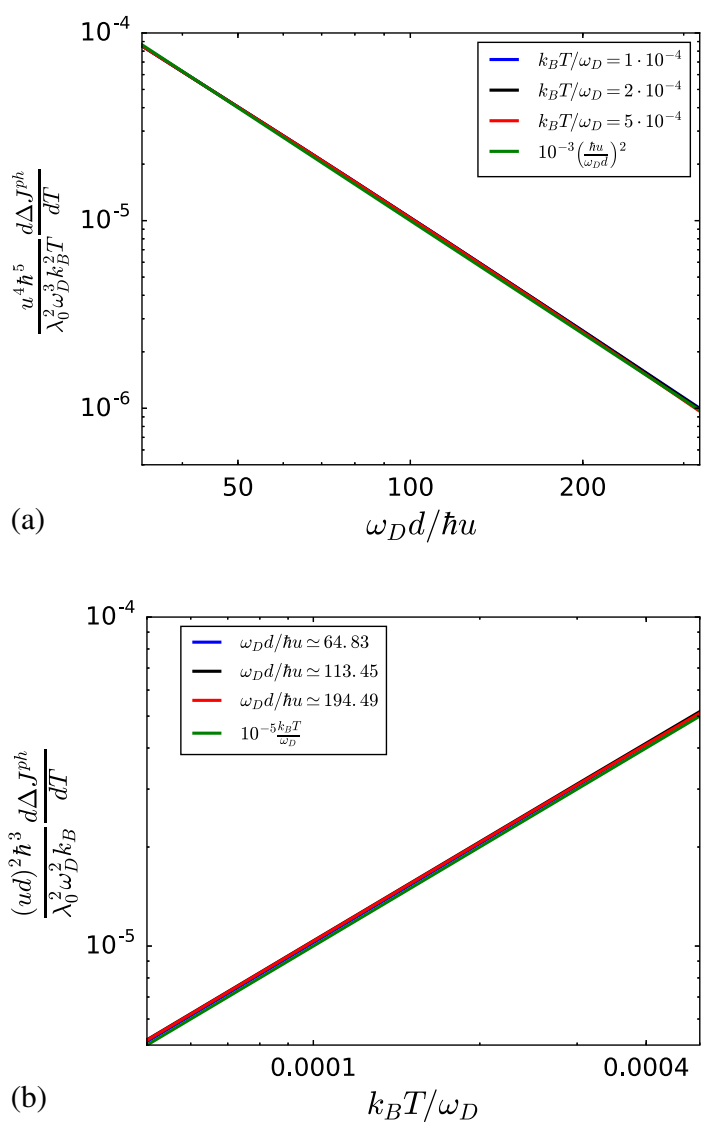

FIG. 4. Dependence of the heat current flowing in the perpendicular direction on (a) the distance $d$ from the edge and (b) the temperature. Here, we plot the temperature derivative of the difference between the heat current for an infinite system and the heat current when considering only a slab of width $d$, for different temperatures in (a) and different distances in (b). The green line shows (a) $1 / d^{2}$ behavior and (b) linear $T$ behavior.

which we believe does not affect the results qualitatively, and we evaluate the temperature derivative of this quantity $d \Delta J_{x}^{\mathrm{ph}}(d) / d T$.

The numerical integration shows that the phonon correction has the following scaling behavior,

$$
\Delta J_{x}^{\mathrm{ph}}(d) \sim \frac{\lambda_{0}^{2} \omega_{D}\left(k_{B} T\right)^{2}}{(u d)^{2} \hbar^{3}},
$$

and, therefore, vanishes for large $d, \Delta J_{x}^{\mathrm{ph}}(d) \propto 1 / d^{2}$; see Fig. 4. This shows that, at least to order $\lambda^{2}$ and in the thermodynamic limit, the edge anomaly is not affected by the coupling to gapless bulk phonons; see the main text.

[1] N. Read and D. Green, Paired States of Fermions in Two Dimensions with Breaking of Parity and Time-Reversal Symmetries and the Fractional Quantum Hall Effect, Phys. Rev. B 61, 10267 (2000).
[2] Z. Wang, X.-L. Qi, and S.-C. Zhang, Topological Field Theory and Thermal Responses of Interacting Topological Superconductors, Phys. Rev. B 84, 014527 (2011).

[3] M. Stone, Gravitational Anomalies and Thermal Hall Effect in Topological Insulators, Phys. Rev. B 85, 184503 (2012).

[4] A. Kitaev, Anyons in an Exactly Solved Model and Beyond, Ann. Phys. (N.Y.) 321, 2 (2006).

[5] Y. Kasahara, T. Ohnishi, N. Kurita, H. Tanaka, J. Nasu, Y. Motome, T. Shibauchi, and Y. Matsuda, Majorana Quantization and Half-Integer Thermal Quantum Hall Effect in a Kitaev Spin Liquid, Nature (London) 559, 227 (2018).

[6] J. Nasu, M. Udagawa, and Y. Motome, Vaporization of Kitaev Spin Liquids, Phys. Rev. Lett. 113, 197205 (2014).

[7] K. W. Plumb, J. P. Clancy, L. J. Sandilands, V. V. Shankar, Y. F. Hu, K. S. Burch, H.-Y. Kee, and Y.-J. Kim, $\alpha-\mathrm{RuCl}_{3}: A$ Spin-Orbit Assisted Mott Insulator on a Honeycomb Lattice, Phys. Rev. B 90, 041112 (2014).

[8] H.-S. Kim, Vijay Shankar V., A. Catuneanu, and H.-Y. Kee, Kitaev Magnetism in Honeycomb $\mathrm{RuCl}_{3}$ with Intermediate Spin-Orbit Coupling, Phys. Rev. B 91, 241110 (2015).

[9] R. D. Johnson, S. C. Williams, A. A. Haghighirad, J. Singleton, V. Zapf, P. Manuel, I. I. Mazin, Y. Li, H. O. Jeschke, R. Valentí, and R. Coldea, Monoclinic Crystal Structure of $\alpha-\mathrm{RuCl}_{3}$ and the Zigzag Antiferromagnetic Ground State, Phys. Rev. B 92, 235119 (2015).

[10] J. A. Sears, M. Songvilay, K. W. Plumb, J. P. Clancy, Y. Qiu, Y. Zhao, D. Parshall, and Y.-J. Kim, Magnetic Order in $\alpha-\mathrm{RuCl}_{3}$ : A Honeycomb-Lattice Quantum Magnet with Strong Spin-Orbit Coupling, Phys. Rev. B 91, 144420 (2015).

[11] L. J. Sandilands, Y. Tian, K. W. Plumb, Y.-J. Kim, and K. S. Burch, Scattering Continuum and Possible Fractionalized Excitations in $\alpha-\mathrm{RuCl}_{3}$, Phys. Rev. Lett. 114, 147201 (2015).

[12] L. J. Sandilands, Y. Tian, A. A. Reijnders, H.-S. Kim, K. W. Plumb, Y.-J. Kim, H.-Y. Kee, and K. S. Burch, Spin-Orbit Excitations and Electronic Structure of the Putative Kitaev Magnet $\alpha-\mathrm{RuCl}_{3}$, Phys. Rev. B 93, 075144 (2016).

[13] L. J. Sandilands, C. H. Sohn, H. J. Park, S. Y. Kim, K. W. Kim, J. A. Sears, Y.-J. Kim, and T. W. Noh, Optical Probe of Heisenberg-Kitaev Magnetism in $\alpha-\mathrm{RuCl}_{3}$, Phys. Rev. B 94 , 195156 (2016).

[14] A. Banerjee, C. A. Bridges, J.-Q. Yan, A. A. Aczel, L. Li, M. B. Stone, G. E. Granroth, M. D. Lumsden, Y. Yiu, J. Knolle, S. Bhattacharjee, D. L. Kovrizhin, R. Moessner, D. A. Tennant, D. G. Mandrus, and S. E. Nagler, Proximate Kitaev Quantum Spin Liquid Behaviour in a Honeycomb Magnet, Nat. Mater. 15, 733 (2016).

[15] X. Zhou, H. Li, J. A. Waugh, S. Parham, H.-S. Kim, J. A. Sears, A. Gomes, H.-Y. Kee, Y.-J. Kim, and D. S. Dessau, Angle-Resolved Photoemission Study of the Kitaev Candidate $\alpha-\mathrm{RuCl}_{3}$, Phys. Rev. B 94, 161106 (2016).

[16] A. Koitzsch, C. Habenicht, E. Müller, M. Knupfer, B. Büchner, H. C. Kandpal, J. van den Brink, D. Nowak, A. Isaeva, and Th. Doert, $J_{\text {eff }}$ Description of the Honeycomb Mott Insulator $\alpha-\mathrm{RuCl}_{3}$, Phys. Rev. Lett. 117, 126403 (2016).

[17] S. Sinn, C. H. Kim, B. H. Kim, K. D. Lee, C. J. Won, J. S. Oh, M. Han, Y. J. Chang, N. Hur, H. Sato, B.-G. Park, C. Kim, H.-D. Kim, and T. W. Noh, Electronic Structure of the 
Kitaev Material $\alpha-\mathrm{RuCl}_{3}$ Probed by Photoemission and Inverse Photoemission Spectroscopies, Sci. Rep. 6, 39544 (2016).

[18] M. Ziatdinov, A. Banerjee, A. Maksov, T. Berlijn, W. Zhou, H. B. Cao, J.-Q. Yan, C. A. Bridges, D. G. Mandrus, S. E. Nagler, A. P. Baddorf, and S. V. Kalinin, Atomic-Scale Observation of Structural and Electronic Orders in the Layered Compound $\alpha-\mathrm{RuCl}_{3}$, Nat. Commun. 7, 13774 (2016).

[19] H. B. Cao, A. Banerjee, J.-Q. Yan, C. A. Bridges, M. D. Lumsden, D. G. Mandrus, D. A. Tennant, B. C. Chakoumakos, and S. E. Nagler, Low-Temperature Crystal and Magnetic Structure of $\alpha-\mathrm{RuCl}_{3}$, Phys. Rev. B 93, 134423 (2016).

[20] H.-S. Kim and H.-Y. Kee, Crystal Structure and Magnetism in $\alpha-\mathrm{RuCl}_{3}$ : An Ab Initio Study, Phys. Rev. B 93, 155143 (2016).

[21] R. Yadav, N. A. Bogdanov, V. M. Katukuri, S. Nishimoto, J. van den Brink, and L. Hozoi, Kitaev Exchange and FieldInduced Quantum Spin-Liquid States in Honeycomb $\alpha-\mathrm{RuCl}_{3}$, Sci. Rep. 6, 37925 (2016).

[22] J. Chaloupkaand G. Khaliullin, Magnetic Anisotropy in the Kitaev Model Systems $\mathrm{Na}_{2} \mathrm{IrO}_{3}$ and $\mathrm{RuCl}_{3}$, Phys. Rev. B 94 , 064435 (2016).

[23] J. Nasu, J. Knolle, D. L. Kovrizhin, Y. Motome, and R. Moessner, Fermionic Response from Fractionalization in an Insulating Two-Dimensional Magnet, Nat. Phys. 12, 912 (2016).

[24] S. Trebst, Kitaev Materials, arXiv:1701.07056.

[25] D. Hirobe, M. Sato, Y. Shiomi, H. Tanaka, and E. Saitoh, Magnetic Thermal Conductivity Far above the Néel Temperature in the Kitaev-Magnet Candidate $\alpha-\mathrm{RuCl}_{3}$, Phys. Rev. B 95, 241112 (2017).

[26] C. Hickey and S. Trebst, Gapless Visons and Emergent U(1) Spin Liquid in the Kitaev Honeycomb Model: Complete Phase Diagram in Tilted Magnetic Fields, arXiv:1805.05953.

[27] A. Banerjee, P. Lampen-Kelley, J. Knolle, C. Balz, A. A. Aczel, B. Winn, Y. Liu, D. Pajerowski, J. Yan, C. A. Bridges, A. T. Savici, B. C. Chakoumakos, M. D. Lumsden, D. A. Tennant, R. Moessner, D. G. Mandrus, and S. E. Nagler, Excitations in the Field-Induced Quantum Spin Liquid State of $\alpha-\mathrm{RuCl}_{3}$, npj Quantum Mater. 3, 8 (2018).

[28] J. M. Luttinger, Theory of Thermal Transport Coefficients, Phys. Rev. 135, A1505 (1964).

[29] L. Alvarez-Gaume and E. Witten, Gravitational Anomalies, Nucl. Phys. B234, 269 (1984).

[30] A. Cappelli, M. Huerta, and G. R. Zemba, Thermal Transport in Chiral Conformal Theories and Hierarchical Quantum Hall States, Nucl. Phys. B636, 568 (2002).

[31] A. P. Schnyder, S. Ryu, A. Furusaki, and A. W. W. Ludwig, Classification of Topological Insulators and Superconductors in Three Spatial Dimensions, Phys. Rev. B 78, 195125 (2008).
[32] S. Ryu, A. P. Schnyder, A. Furusaki, and A. W. W. Ludwig, Topological Insulators and Superconductors: Tenfold Way and Dimensional Hierarchy, New J. Phys. 12, 065010 (2010).

[33] S. Ryu, J. E. Moore, and A. W. W. Ludwig, Electromagnetic and Gravitational Responses and Anomalies in Topological Insulators and Superconductors, Phys. Rev. B 85, 045104 (2012).

[34] K. Nomura, S. Ryu, A. Furusaki, and N. Nagaosa, CrossCorrelated Responses of Topological Superconductors and Superfluids, Phys. Rev. Lett. 108, 026802 (2012).

[35] A. Gromov and A. G. Abanov, Thermal Hall Effect and Geometry with Torsion, Phys. Rev. Lett. 114, 016802 (2015).

[36] M. Banerjee, M. Heiblum, V. Umansky, D. E. Feldman, Y. Oreg, and A. Stern, Observation of Half-Integer Thermal Hall Conductance, arXiv:1710.00492.

[37] M. Banerjee, M. Heiblum, A. Rosenblatt, Y. Oreg, D. E. Feldman, A. Stern, and V. Umansky, Observed Quantization of Anyonic Heat Flow, Nature (London) 545, 75 (2017).

[38] K. Sugii, M. Shimozawa, D. Watanabe, Y. Suzuki, M. Halim, M. Kimata, Y. Matsumoto, S. Nakatsuji, and M. Yamashita, Thermal Hall Effect in a Phonon-Glass $\mathrm{Ba}_{3} \mathrm{CuSb}_{2} \mathrm{O}_{9}$, Phys. Rev. Lett. 118, 145902 (2017).

[39] C. Strohm, G. L. J. A. Rikken, and P. Wyder, Phenomenological Evidence for the Phonon Hall Effect, Phys. Rev. Lett. 95, 155901 (2005).

[40] L. Sheng, D. N. Sheng, and C.S. Ting, Theory of the Phonon Hall Effect in Paramagnetic Dielectrics, Phys. Rev. Lett. 96, 155901 (2006).

[41] J.-S. Wang and L. Zhang, Phonon Hall Thermal Conductivity from the Green-Kubo Formula, Phys. Rev. B 80, 012301 (2009).

[42] L. Zhang, J. Ren, J.-S. Wang, and B. Li, Topological Nature of the Phonon Hall Effect, Phys. Rev. Lett. 105, 225901 (2010).

[43] T. Qin, J. Zhou, and J. Shi, Berry Curvature and the Phonon Hall Effect, Phys. Rev. B 86, 104305 (2012).

[44] M. Mori, A. Spencer-Smith, O. P. Sushkov, and S. Maekawa, Origin of the Phonon Hall Effect in Rare-Earth Garnets, Phys. Rev. Lett. 113, 265901 (2014).

[45] H. B. G. Casimir, Note on the Conduction of Heat in Crystals, Physica (Utrecht) 5, 495 (1938).

[46] S. Rachel, L. Fritz, and M. Vojta, Landau Levels of Majorana Fermions in a Spin Liquid, Phys. Rev. Lett. 116, 167201 (2016).

[47] T. Qin, Q. Niu, and J. Shi, Energy Magnetization and the Thermal Hall Effect, Phys. Rev. Lett. 107, 236601 (2011).

[48] M. Ye, G. B. Halász, L. Savary, and L. Balents, Quantization of the Thermal Hall Conductivity at Small Hall Angles, arXiv:1805.10532. 